\title{
A Survey on Feedback Session for Inferring User Search Query with CAP
}

\author{
Shweta K. Sirse' ${ }^{1}$ C. S. Biradar ${ }^{2}$ \\ ${ }^{1}$ SRTM University, Department of CSE, M. S. Bidve Engineering College, Latur, Maharashtra, India, \\ ${ }^{2}$ Visvesvaraya Technological University, Department of CSE, B.E.C. College of Engineering, Bagalkot, Karnataka, India
}

\begin{abstract}
Data mining is extracting or mining knowledge from large amount of data it is called knowledge mining from data information searching is one of the important phenomenon in today's world .users prefer to search to by their query to explain their recognized uncertain in sequence search engines does not give correct result of user required query and does not satisfy the request hence it is necessary to infer and analysis user specific interest about query. The inference and analysis of user search goals can be useful in improving performance of search engine. inferring and analysis of user search goals is proposed in this paper .this paper proposes new method to infer user search goals by analyzing search engine queries. we propose 2 different framework.first we propose framework to find out different user search information for user query by using clustering the proposed feedback session .feedback session buit/ construct from user click through logs consist the information about different user search information. Using pseudo document the feedback session are represented second method to generate pseudo document to represent the feedback sessions for clustering for pseudo document clustering we use k-means clustering algorithm .finally to calculate the performance of user search goal inference we use CAP(classified average precision) algorithm.CAP is useful in reconstructing search engine performance.
\end{abstract}

Keywords: CAP (Classified average precision),Feedback sessions, Pseudo documents, user search query, knowledge mining

\section{Introduction}

Web mining is the data mining techniques to automatically discover and extract information from web documents and services. collection of connected files on one or more web servers known as web .data mining application is web mining.web data consist text ,image, record of web content data, web structure data, web usage data .web usage data consist Http log and domestic logs. in the network search, users are suggested queries to the search engines to get important data. but some search engines failed to produce user data results. different users are usually search ambiguous keywords representing user interest .unclear keywords do not match with search engine result. According to web search application and the user search goals the related work is produce in earlier clustering is done on sequence of ranked results .K-means clustering algorithm used for clustering the pseudo documents. To cluster user search results URL'S are analyzed by mining the data.

In this paper we use the web procedure mining information. network server registers a data entry for accessing web page. it consist the URL requested the IP address .in which the request originated and time stamp based on the web log records. for these construct feedback session because web log data provide the information about which kind of users will access the web pages. Pseudo document produced by using feedback session to evaluate correct result. pseudo documents which consist of URLS keywords in feedback session this called enriched URLs. by using enriched URLs clustering the pseudo document is formed. clustering is the process of grouping the similar data into dissimilar data object.

After pseudo document process the web search results are reproduced. when more insignificant and significant data are produced by the user search engine it is time consuming to search. the user submit the query to search engine or user browser the search engine .the search engines checks the significant data related to user query. User data saved in the user click through logs. Feedback session produced and reviewed by using user click through logs. Feedback session consist URLS and the click sequence. Pseudo documents consist the keys that are clicked in a session. the CAP evaluation can be done for user search query and the clustering done to search different no of users. Clustering explore outcome is an proficient technique to arrange search results, which allow a user to search the method into appropriate papers quickly. In this paper, our aim is to determine dissimilar customer search goals for a query and represent each search aim with some keywords repeatedly.

\section{Web Usage Mining}

Web usage mining which mines the weblog records to determine user access patterns of web pages. in developing techniques for web usage mining consider the following the URL,time,IP address and web page content information multidimensional view can be construct on the weblog record and multidimensional OLAP analysis can be produced to identify top $\mathrm{N}$ users. data mining can be performed on weblog records to identify association model, sequential model, and things of web accessing. Clustering is process of grouping similar data. we make use of $\mathrm{k}$ means clustering method for construct pseudo documents.

\section{Related Work}

The problem of clustering examine results has been study in a number of earlier work. the earlier work submit clustering algorithm in which $1^{\text {st }}$ group documents into same group 


\section{International Journal of Science and Research (IJSR) \\ ISSN (Online): 2319-7064}

Index Copernicus Value (2013): 6.14 | Impact Factor (2014): 5.611

equal to content of similar data and create important analysis for cluster.

\subsection{Suffix Tree Clustering}

Zamir and Etzioni[9]proposed a suffix tree clustering (STC).initially introduces information of data documents that divides common phrases and after dividing common phrases that produce clusters According to phrases. the phrase extraction procedure is same to suffix tree clustering .network search is a challenge to provide users information needs by using web pages with reverence to user query. series of interface amongst user and search engine be able to necessary to convince a solitary in order need.

\subsection{User Search task for query}

Though users query search engines in order to achieve tasks at a diversity of granularities, issue numerous query as they effort to accomplish tasks .R Jones and K.L linker[5][1]learning real sessions manually labeled into hierarchical tasks and demonstrate that timeout, anything their length are of incomplete utility in identifying task boundaries, achieving greatest precision their method only identifies whether bunch of queries related to similar goal and does not mind what the goal is in aspect

\subsection{Automated identification of user goals in network search engine.}

U.Lee ,Z.Liu and J.cho[6] study the ,goale at the behind based on user"s query .so that this goal used to get better the exceuence of a investigate engine"s results.

Manual query log analysis were used to recognize the user goals in existing system[4].proposed system consist automatic goal identification process.

Classification of query based on 2types.

\section{1) Navigating query}

In this user set the specified web page and initially visit the specified web page user might also have visit that site before, or just assume such a place exists. Useres will only visit the accurate sites.

\section{2) Instructional query}

Instructional / Informational queries are the queries where user does not have particular page in mind to visit multiple pages to learn about the subject.user search the web pages that provide information about specified query .users click on multiple results because they do not suppose particular website to be single correct answer

\subsection{Optimizing search engines using click through data}

T.Joachims in 2002 presents an approach for automatically optimizing the retrieval quality of search engines using click through data. Good data retrieval system should present documents high in the ranking, with less relevant documents.
T.Joachims evaluate approaches for how to mechanically develop training data example for study of retrieval functions from experiments. still absolute feedback is tough to explain and theoretically noisy. Initially study which types of definite feedback can be removed from experiential user, in click through data i.e WWW network search. to evaluate the reliability of definite feedback signals to study how users correspondence with the list of ranked sequences from search engine. Thorsten Joachimes works on how to definite feedback to get good quality.

In our new approach feedback sessions as user implicit feedback and suggest a novel optimization method to merge both clicked and unlocked URL's in feedback sessions to discover what users really necessary and what they do not mind

\subsection{Generating query substitutions/Producing query replacement}

Query replacement produce the new query to change the useres original query. this method uses modification based on query substitution the another queries and the name are connected to the new queries.

Query replacement is inverse with query extension and query reduction.

The query extension gives the pseudo document feedback. The query recreation is using Boolean or TF-IDF and it reduces query specification.

Calculation of user query replacement produce well at evaluation of new queries compared to original user queries. Experiments present that this method automatically raises coverage and effectiveness in the network search.

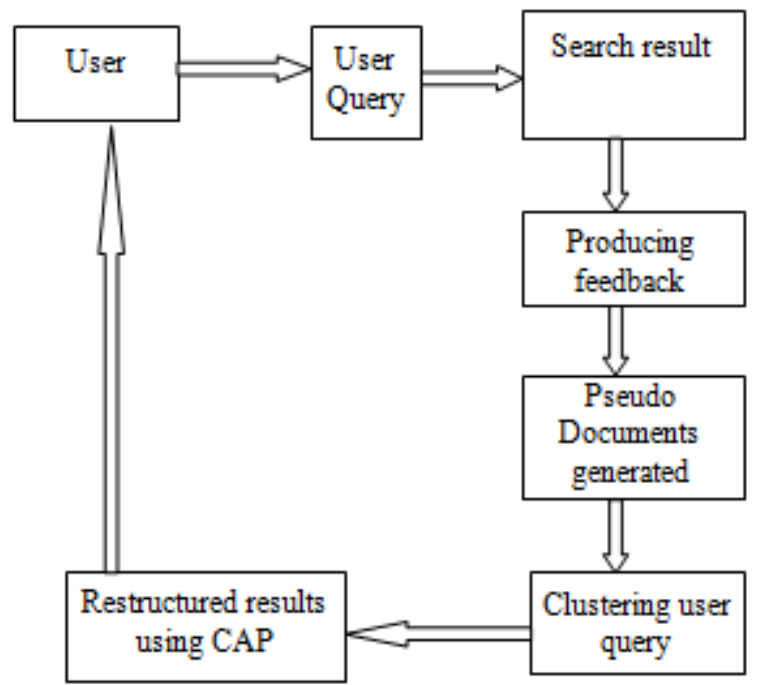

Figure 1: System Architecture

\subsection{Learn from web search logs to organize search results.}

To organize search results learn from web search goals.

Search engines results of the efficient organization are hard to improve the efficiency of the search engine.

Search results clustering is the best way to form the results. 


\section{International Journal of Science and Research (IJSR)}

ISSN (Online): 2319-7064

Index Copernicus Value (2013): 6.14 | Impact Factor (2014): 5.611

There are 2 errors.

1)The clusters do not trust on the aspects of users.

2)The cluster tags are not useful, so the correct cluster identification is hard.

For these reasons are

1)Tags are not meaningful

2)Tags are not informative

Solution for the errors in proposed

1)Learning ,Important things ${ }^{\text {ee }}$ from network search goals and producing search results.

2)Informative cluster tags are produced using query words of user. By using session the user search engine logs separated. Session consist a single user query and all user URL"s.

\subsection{Study query determination from fixed click graph.}

Improve the query determination by using click graphs. Method is hard for user general purpose search engines. The important objective is to improve amount of data by using supervised study with click graphs. Based on the click graph, unnamed queries are produced from labeled queries. Moreover the study with clicked graphs by using classification for the avoidance of error labels.

\section{Different applications are used}

First product intent, $2^{\text {nd }}$ job intent classification by using this method training data can be expressed and adds to improvements in classification.

\subsection{Clustering web search results.}

Q-C He ,Z.chen,H-J Zeng,W-Y ma,J ma(4) researched on reproducing the clustering.

Approach consist following 4 steps

1) Fetching the search result

2) Text parsing and phrase characteristic calculation.

3) Salient expression ranking/phrase ranking

4) Post refinement

Query and search results list are given .initially the complete list of labels is parsed,extrats the all phrases from index and count several properties of phrase.

Then the regression method applied to connect properties into single salience score. According to phrase ranking score the phrases ranked and the top phrases ranked and the top phrases is select as phrase salient, post refinement, filter out the pure stop words.

\section{Conclusion}

In this paper, a new approach has been proposed to infer user search goals for a uncertainty by using clustering its feedback session. initial, we introduce feedback sessions to be analyzed to infer user search goals relatively than look for results. Both the clicked URLs and the unclicked ones before the last click are consider as client implied feedbacks and it taken into account to construct feedback sessions.
Therefore, feedback sessions can replicate user in sequence requirements more efficiently. Second, we map feedback sessions to pseudo documents to inexact aim texts in consumer minds. The pseudo-documents can enhance the URLs with additional textual inside including the title and odds and ends. Based on these, user searh goals can then be exposed and depict with some keywords. lastly, a new criterion CAP(Classified Average Precision) is formulate to estimate the performance of user search goal inference. new results on user click-through logs from a viable search engine display the efficiency of our proposed methods. The difficulty of our advance is low and our approach can be used in actuality simply. For query, the operation time depends on the numeral of feedback sessions. However, the dimension of Ffs in (3) and (5) is not very high. Therefore, the operation time is usually short. our progress can determine user search goals for some popular queries offline at. Then, when users propose queries, the search engine can return the results that are categorized into different groups . Thus, users can find what they want conveniently

\section{References}

[1] L.Suganya,Dr.B.Srinivasan"Efficient Semantic Similarity Based FCM For Inferring User Search Goals With Feedback Sessions.'International Journal Of Computer Trends and Technology(IJCTI)-vol 4Issue 9sep2013.

[2] D.Kavitha,K.M.Subramanian,Dr.K.Venkatachalam "Survey On Inferring User Search Goal Using Feedback Session”.ISSN:2278-1323,IJARCET vol 2,December 2013.

[3] R.Dhivya,R.Rajavignesh "A Novel Approach For Restructuring Web Search Results By Feedback Sessions Using Fuzzy Clustering”.International Journal Of Innovative Reaserch In Computer And Communcation Engineering.vol 2,special Issue1,March 2014.

[4] Sukanya S.Gawade,Gyankamal J.Chhajed "Using Feedback Sessions For Inferring User Search Goals"International Journal Of Advanced Research In Computer And Communication Engineering.vol 3,Issue 6,June 2014.

[5] R.Jones and K.L.Klinker, "Beyond The Session Timeout: Automatic Hierarchical Segmentation of Search Topics In Query logs."Proc. $17^{\text {th }}$ ACM Conf.International And knowledge Management,pp.699708,2008. Approaches" . In z. Karakehayov.: data Acquisition applications: cha.13,2012.

[6] U.Lee,Z.Liu,and J.Cho, "Automatic Identification Of User Goals In Web Search,"Proc.14 ${ }^{\text {th }}$ International 1 conf.World Wide Web,pp.391-400,2005.

[7] T.Joachims, "Evaluating Retrieval Performance UsingClickthroughData,'Text

Mining,J.Franke,G.Nakhaeizadeh,and I.Renz,eds,pp 7996,2003

[8] Charudatt Mane, Pallavi Kulkarni "A Novel Approach to Discover User Search Goals Using Clickthrough Data" Charudatt Mane et al, / (IJCSIT) International Journal of Computer Science and Information Technologies, Vol. 5 (1) , 2014, 20-24. 
[9] Ms. S. S. Jadhav, Prof. N. D. Kale "Improved Accuracy and User Satisfaction by Inferring User Search Goals based on Feedback Sessions" S. S. Jadhav et al, International Journal of Computer Science and Mobile Computing, Vol.3 Issue.6, June- 2014, pg. 184-192.

[10] Agale Deepali B,Prof.B.S.Khade "Inferring User Search Goals Engine" International Journal Of Advancements In Research \&Technology, Vol 3,Issue 6,June-2014.

\section{Author Profile}

S. K. Sirse received the B.E. degree in Computer Science and Engineering from M.S. Bidve Engineering College in 2013. Now, she is pursuing Master"es in Engineering (Computer Science and Engineering) from M.S.Bidve Engineering college, Latur ,SRTM University Nanded, Maharashtra.

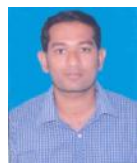

Prof. C. S. Biradar received the B.E. and M.Tech. degrees in Computer Science \& Engineering from B. L. D. E. A College of Engineering, Bijapur in 2011 and from BEC College of Engineering, Bagalkot in 2013, respectively. He is now with M.S. Bidve Engineering College, Latur(M.S.) as Assistant Professor since 2013. 